\title{
Gupta Transform Approach to the Series RL and RC Networks with Steady Excitation Sources Rahul Gupta $^{*}{ }^{1}$, Rohit Gupta ${ }^{\# 2}$, Loveneesh Talwar ${ }^{\# 3}$ \\ ${ }^{1,2}$ Lecturer of Physics, Department of Applied Sciences, Yogananda College of Engineering and Technology, Jammu, J\&K, India ${ }^{3}$ Assistant Professor, Department of Electrical Engineering, Yogananda College of Engineering and Technology, Jammu, J\&K, India
}

\begin{abstract}
The analysis of electric networks circuits is an essential course in engineering. The response of such networks is usually obtained by mathematical approaches such as Laplace Transform, Calculus Approach, Convolution Theorem Approach, Residue Theorem Approach. This paper presents a new integral transform called Gupta Transform for obtaining the complete response of the series RL and RC networks circuits with a steady voltage source. The response obtained will provide electric current or charge flowing through series RL and RC networks circuits with a steady voltage source. In this paper, the response of the series RL and RC networks circuits with steady excitation source is provided as a demonstration of the application of the new integral transform called Gupta Transform.
\end{abstract}

Keywords - Gupta Transform; Series RL and RC Networks circuits; Response.

\section{Introduction}

The electric circuit of the series RL network consists of two passive electric elements: an inductor $€$ and a resistor $R$, connected in series with asteady voltage source and theelectric circuit of the series RC network consists of two passive electric elements: a capacitor $\mathrm{C}$ and a resistor $\mathrm{R}$, connected in series with a steady current source. Such networks are used as a tuning or resonant circuit in the radio and television set to a particular frequency band from the wide range of frequency components, or in the chokes of luminescent tubes [1-4]. The Gupta Transform has been proposed recently by the authors Rahul Gupta and Rohit Gupta and generally, it has been applied in different areas of science and engineering $[5,6]$.The response of electrical networks is generally obtained by the different mathematical approaches like the calculus approach [1-3], convolution theorem approach [7], or by various integral transforms like Laplace Transform [1-3], Mohand Transform [8, 9], Aboodh Transform [10], Elzaki Transform [11], residue theorem approach [12], Rohit Transform [13]. This paper presents the use of a new integral transform called Gupta Transform for obtaining the complete response of the series RL and RC networks circuits with a steady voltage source and brings up the Gupta Transform as a new successful powerful tool for determining the response of network circuits.

\section{Gupta Transform}

Let $\mathrm{g}(\mathrm{y})$ is a continuous function on any interval for $\mathrm{y} \geq$ 0 . The Gupta Transform of $g(y)$ is as $[5,6]$
$\dot{\mathrm{R}}\{\mathrm{g}(\mathrm{y})\}=\frac{1}{q^{3}} \int_{0}^{\infty} e^{-q y} g(y) d y=G(q)$, provided that the integral is convergent, where, $q$ may be a real or complex parameter and $\dot{\mathrm{R}}$ is the Gupta Transform operator. The Gupta Transform of elementary functions are given in $[5,6,15]$. The inverse Gupta Transform of the function $G(r)$ is denoted by $\dot{\mathrm{R}}^{-1}\{\mathrm{G}(\mathrm{r})\}$ or $\mathrm{g}(\mathrm{y})$. If we write $\dot{\mathrm{R}}\{\mathrm{g}(\mathrm{y})\}=\mathrm{G}(\mathrm{r})$, then $\dot{\mathrm{R}}^{-}$ ${ }^{1}\{\mathrm{G}(\mathrm{r})\}=\mathrm{g}(\mathrm{y})$, where $\dot{\mathrm{R}}^{-1}$ is called the inverse Gupta Transform operator. The Inverse Gupta Transform of elementary functions are given in $[5,6]$. The Gupta Transform of some derivatives $[5,6,15]$ of $\mathrm{g}(\mathrm{y})$ are,

$$
\begin{gathered}
\dot{\mathrm{R}}\left\{g^{\prime}(y)\right\}=q \dot{\mathrm{R}}\{g(y)\}-\frac{1}{q^{3}} g(0), \\
\dot{\mathrm{R}}\left\{g^{\prime \prime}(y)\right\}=q^{2} \dot{\mathrm{R}}\{g(y)\}-\frac{1}{q^{2}} g(0)-\frac{1}{q^{3}} g^{\prime}(0) .
\end{gathered}
$$

\subsection{Series RI Network Circuit with Steady Voltage Source}

We will take a series RL network circuit to which a steady voltage source of potential $V_{0}$ is applied through a key $\mathrm{K}$ as shown in figure 1 .

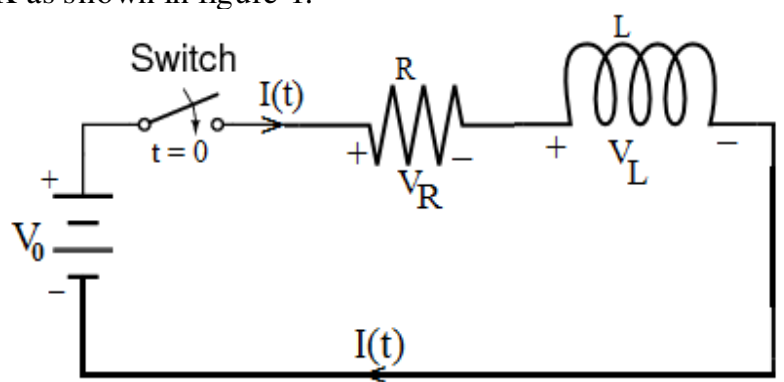

Fig.1: Series RL network with steady voltage source

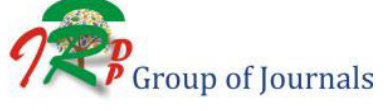


As the switch is closed at $\mathrm{t}=0$, the potential drops across the network elements are given by [1-4].

$V_{\mathrm{R}}(\mathrm{t})=\mathrm{I}(\mathrm{t}) \mathrm{R}, \mathrm{V}_{\mathrm{L}}(\mathrm{t})=\mathrm{tI} \mathrm{I}^{\prime}(\mathrm{t})$.

Therefore, the application of Kirchhoff's loop law to the loop shown in figure 2 provides

$V_{R}(t)+V_{t}(t)=V$

\section{Or}

$\mathrm{R} \mathrm{I}(\mathrm{t})+\left\llcorner\left[\mathrm{I}^{\prime}(\mathrm{t})\right]=\mathrm{V}_{0}\right.$

Differentiating (1), a differential equation of order 2 as given below:

$$
I^{\prime \prime}(t)+\frac{\mathrm{R}}{\mathrm{t}} I^{\prime}(t)=0
$$

To solve equation (2), we first write the in itial conditions as follows [1-4]:

- Since the current in the inductor and the electric potential difference across the capacitor cannot change instantaneously, therefore, as the switch is closed at the instant $\mathrm{t}=0$, then $\mathrm{I}(0)=0$.

- Since $I(0)=0$, therefore, $(1)$ provides $\left[I^{\prime}(0)\right]=\frac{v_{0}}{t}$.

The Gupta Transform [5, 6, 15] of (2) provides

$q^{2} \bar{I}(\mathrm{q})-\frac{1}{q^{2}} I(0)-\frac{1}{q^{3}} I^{\prime}(0)+\frac{\mathrm{R}}{\mathrm{E}}\left\{q \bar{I}(\mathrm{q})-\frac{1}{q^{3}} I(0)\right\}=0$

Applying $I(0)=0$ and $\left[\mathrm{I}^{\prime}(0)\right]=\frac{\mathrm{V}_{0}}{\succeq},(3)$ becomes,

$q^{2} \bar{I}(\mathrm{q})-\frac{1}{q^{3}} \frac{\mathrm{v}_{0}}{\mathrm{t}}+\frac{\mathrm{R}}{\mathrm{q}} q \bar{I}(\mathrm{q})=0$

Or

$\bar{I}(\mathrm{q})=\frac{\mathrm{v}_{0}}{\mathrm{t}}\left[\frac{1}{q^{3}} \frac{1}{q\left(q+\frac{\mathrm{R}}{\mathrm{E}}\right)}\right]$

This equation can be rewritten as

$\bar{I}(\mathrm{q})=\frac{\mathrm{V}_{0}}{\mathrm{R}}\left[\frac{1}{q^{4}}-\frac{1}{q^{3}} \frac{1}{q+\frac{\mathrm{R}}{\mathrm{t}}}\right]$

Taking inverse Gupta Transform [5, 6], we have

$I(t)=\frac{\mathrm{V}_{0}}{\mathrm{R}}\left[1-e^{-\left(\frac{\mathrm{R}}{\mathrm{E}}\right) t}\right]$

This equation (5) provides the complete response of the series RL network circuit with a steady potential source.

\subsection{Series RC Network Circuit with Steady Voltage Source}

The series R-C network circuit with a steady potential source (as show in figure 2) is analyzed by the following equation [1-3]

$\dot{\mathrm{Q}}(\mathrm{t}) \mathrm{R}+\frac{\mathrm{Q}(\mathrm{t})}{\mathrm{C}}=\mathrm{V}_{0}$

Or

$\dot{\mathrm{Q}}(\mathrm{t})+\frac{1}{\mathrm{RC}} \mathrm{Q}(\mathrm{t})=\frac{\mathrm{V}_{0}}{R}$
Here, $Q(t)$ is the instantaneous charge and $Q(0)=0$.

The Gupta Transform [5, 6, 15] of (6) gives

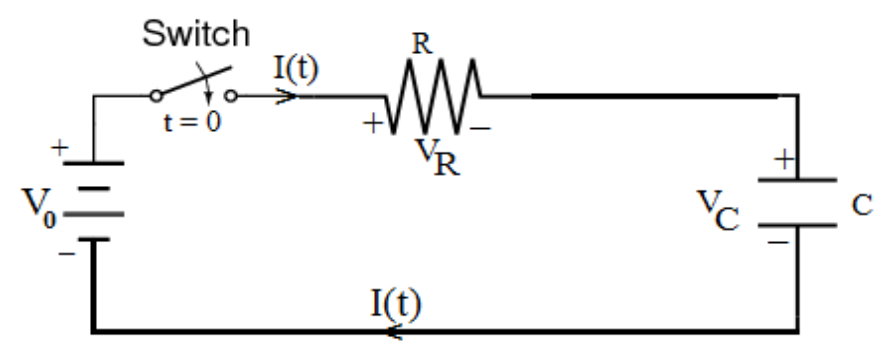

Fig.2: Series RC network with steady voltage source

$\left\{q \bar{Q}(\mathrm{q})-\frac{1}{q^{3}} Q(0)\right\}+\frac{1}{\mathrm{RC}} \bar{Q}(\mathrm{q})=\frac{\mathrm{v}_{0}}{\mathrm{R}}$

Put Q $(0)=0$ we get

$\{q \bar{Q}(\mathrm{q})\}+\frac{1}{\mathrm{RC}} \bar{Q}(\mathrm{q})=\frac{\mathrm{V}_{0}}{\mathrm{R}} \frac{1}{q^{3}}$

This equation can be rewritten as

$\bar{Q}(\mathrm{q})=\frac{\mathrm{V}_{0}}{\mathrm{R}} \frac{1}{q^{3}} \frac{1}{\left(q+\frac{1}{\mathrm{RC}}\right)}$

Or

$\bar{Q}(\mathrm{q})=\mathrm{CV}_{0}\left[\frac{1}{q^{4}}-\frac{1}{q^{3}} \frac{1}{q+\frac{1}{\mathrm{RC}}}\right]$

Taking inverse Gupta Transform [5, 6], we have

$Q(t)=\mathrm{CV}_{0}\left[1-e^{-\left(\frac{1}{\mathrm{RC}}\right) t}\right]$

This equation (8) provides the complete response of the series RC network circuit with a steady potential source.

\section{Conclusion}

In this paper, a new integral transform called Gupta Transform has been successfully applied for determining the complete response of the series RL and RC networks with a steady excitation source. The results obtained are the same as obtained with other approaches [1-3, 14]. This paper brought up the Gupta Transform as a new successful powerful tool for determining the response of network circuits.

\section{References}

[1] Network Analysis M. E. Van Valkenburg. 3rd Edition, Publisher: Pearson Education, 2015.

[2] J. S. Chitode and R.M. Jalnekar, Network Analysis and Synthesis, Publisher: Technical Publications, 2007.

[3] Murray R. Spiegel, Theory and Problems of Laplace Transforms. Publisher: Schaum's out line series, McGraw - Hill.

[4] Rohit Gupta, Rahul Gupta, Sonica Rajput, Convolution Method for the Complete Response of a Series L-R Network Connected to an Excitation Source of Sinusoidal Potential, International Journal of Research in Electronics And Computer Engineering, Vol. 7, issue 1, January- March 2019, pp. 658-661. 
[5] Rahul Gupta, Rohit Gupta, Dinesh Verma, Propounding a New Integral Transform: Gupta Transform with Applications in Science and Engineering, International Journal of Scientific Research in Multidisciplinary Studies, 6(3), March (2020),pp. 14-19.

[6] Rahul Gupta, Rohit Gupta, Dinesh Verma, Application of Novel Integral Transform: Gupta Transform to Mechanical and Electrical Oscillat ors, ASIO Journal of Chemistry, Physics, Mathematics \& Applied Sciences (ASIO-JCPMAS), 2020,4(1): 04-07.

[7] Rohit Gupta, Yuvraj Singh Chib, Rahul Gupta, Design of the resistorcapacit or snubber network for a d. c. circuit containing an inductive load, Journal of Emerging Technologies and Innovative Research (JETIR), Volume 5, Issue 11, November 2018,pp. 68-71.

[8] Rohit Gupta, Anamika Singh, Rahul Gupta, Response of Network Circuits Connected to Exponential Excitation Sources, International Advanced Research Journal in Science, Engineering and Technology, Vol.7, Issue 2, Feb. 2020, pp.14-17.

[9] P. Senthil Kumar,S. Vasuki, Applications of Aboodh Transform to Mechanics, Electrical Circuit Problems, International Journal for Research in Engineering Application \& Management, Vol-04, Issue06, Sep. 2018.

[10] Rohit Gupta and Loveneesh Talwar, Elzaki Transform Means To Design A Protective RC Snubber Circuit, International Journal of Scientific and Technical Advancements, 6(3), 2020: 45-48.

[11] Rahul Gupta and Rohit Gupta, Impulsive Responses of Damped Mechanical and Electrical Oscillators, International Journal of Scientific and Technical Advancements, 2020, 6(3): 41-44.

[12] Rohit Gupta, Loveneesh Talwar, Dinesh Verma, Exponential Excitation Response of Electric Net work Circuits via Residue Theorem Approach, International Journal of Scientific Research in Multidisciplinary Studies, volume 6, issue 3, pp. 47-50, March (2020).

[13] Rohit Gupta, Rahul Gupta, Analysis of RLC circuits with exponential excitation sources by a new integral transform: Rohit Transform, ASIO Journal of Engineering \& Technological Perspective Research (ASIOJETPR), 2020, 5(1): 22-24.

[14] Rohit Gupta, Loveneesh Talwar, Rahul Gupta, Analysis of network circuit with steady voltage source, and with steady current source via convolution method, International Journal Of Scientific \& Technology Research, Volume 8, Issue 11, November 2019, pp. 803-807.

[15] Rahul Gupta, Rohit Gupta, Loveneesh Talwar, Response of Net work Circuits Connected To Impulsive Potential Source via New Integral Transform: Gupta Transform, ASIO Journal of Engineering \& Technological Perspective Research (ASIO-JETPR), 5(1), 2020:18-20. 